\title{
Evaluation of Optical Ring Network to Measure Inter-channel Crosstalk using "Eye Diagrams"
}

\author{
Bhupesh Bhatia ${ }^{1}$, Vijayraj Shokeen ${ }^{1}$ \& R. K. Singh ${ }^{2}$ \\ ${ }^{1}$ Ph.D Scholar-ECE, ${ }^{2}$ ECE Deptt. \\ UTU, Dehradun
}

\begin{abstract}
In this paper we traverse a range over the performance of wide division multiplexing(wdm) optical network connected as the ring topology(ringO network) consist of various nodes has been manifested, and the signal is examined critically as it goes through every node in the network. It has been proved that there is no considerable signal degradation in the ring network. It is seen that the signal keeps on improving as it goes through the consecutive nodes. Also when the entire ring structure is rehearsed with the help of spans, then advancement in the signal is perceived. We investigated the effect of inter-channel crosstalk at ADM of a ring network at sweeping bandwidth of filters and the analysis done using eye diagrams. The effect of increasing the input power is also carried out further and it is seen that it can support more number of users by enhancing the signal input power. The optical spectrum of the signal is observed at each node for assessing of the add and the drop frequencies in the ring.
\end{abstract}

\section{Keywords}

Dense wavelength division multiplexing, Add Drop Multiplexer, Optical Burst Switching, Optical Circuit Switching.

\section{INTRODUCTION}

The optical networks are high-capacity telecom networks based on optical technologies and components; basic need of network is routing, training and reestablishment at the wavelength level and wave-length-based services. The wavelength division multiplexing exhibits the exponential rise in the high speed internet transmission technology. There are many network architectures used for the same reason. The key concluding instruments are adding drop multiplexers (ADM) and Erbium-doped fiber Amplifier (EDFA). Currently, the dense wavelength-division multiplexing (DWDM) technology consummate multiplexing of 150-300 wavelengths in one fiber with 10-40 Gbs transmission rate per wavelength. In a WDM system ADM is used to particularly add and drop any wavelength channel at intermediate node without influencing other channels transmitted synchronously. ADM elevate networks flexibility and capacity as dropped wavelength can be reuse. ADM enhances networks flexibility and capacity as dropped wavelength can be reused. The cost incurred by the sonet ADMs is very high. [1] Optical burst switching (OBS) is a switching technique that covers the middle of the spectrum between the well-known circuit switching and packet switching paradigms, carrying ideas from both to deliver a completely new functionality. OBS is a compromise between optical circuit switching (OCS) and optical packet switching (OPS). The information in OBS nodes is used only locally; thus the network as a whole system does not benefit from the information available on each of the individual nodes. Ring topology is preferred over other network because the number of links is reduced in ring topology. Ring networks (ringO network) provide standby links, share the load and have better resilience. In a ring network, interchannel crosstalk can arise from any of the sources like add-drop multiplexer (ADM), an optical switch, an amplifier, router and XPM [2, 3]. In such cases, a particular signal can accumulate crosstalk from different elements and channels. Some of the key components of optical ring networks are Erbium-doped fiber Amplifier (EDFA) and ADMs. EDFA is a crucial element in optical networks and in an optical or IR repeater that amplifies a modulated beam directly, the need for optoelectronic and electro-optical conversion no longer exist. [4]

\section{SYSTEM DESCRIPTION}

The network topology that is set up in a circular fashion in which data travels around the ring in one direction and each device on the right acts as a repeater to keep the signal strong as it travels. Each device incorporates a receiver for the incoming signal and a transmitter to send the data on to the next device in the ring. The network is dependent on the ability of the signal to travel around the ring.[7]

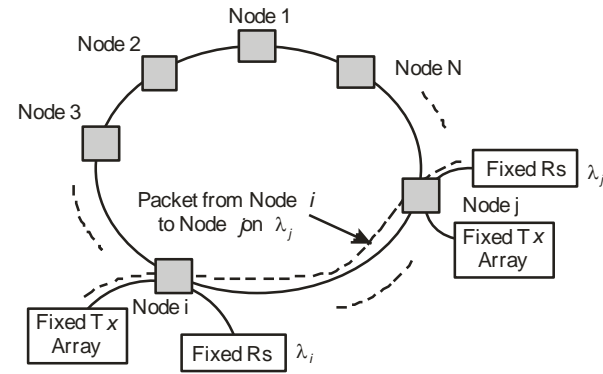

Fig. 1. Ring metro WDM network.

A traditional OADM comprises of following stages: an optical demultiplexer, an optical multiplexer, and between them a technique of reconstructing the paths between the optical demultiplexer, the optical multiplexer and a group of ports for transmitting and receiving signals. The optical demultiplexer separates wavelengths in an input fiber onto ports. The reconfiguration can be achieved by a fiber patch panel or by optical switches which direct the wavelengths to the optical multiplexer or to drop ports. The optical multiplexer multiplexes the wavelength channels that are to continue on from demultipexer ports with those from the add ports, onto a single output fiber. Physically, there are several ways to realize an OADM. There are a variety of demultiplexer and multiplexer technologies including thin film filters, fiber Bragg gratings with optical circulators, free space grating devices and integrated planar Arrayed waveguide gratings. The switching or reconfiguration functions range from the manual fiber patch panel to a variety of switching technologies including MEMS, Liquid crystal and thermo optic switches in planar waveguide circuits.[7] 
This another network contains four nodes that communicate over two channels at 185 and $185.1 \mathrm{THz}$, as shown in Fig. 2. Four nodes are all transceivers and they are interconnected with each other through single mode fibers. All the Transceivers are modeled on fourth-order Bessel filters having $15 \mathrm{dBm}$ sensitivity. Before Node 2 an optical amplifier (EDFA) with gain $12 \mathrm{~dB}$ and noise figure $5 \mathrm{~dB}$ has been included. In this network the bandwidth of three transceivers was kept fixed at $10 \mathrm{GHz}$ and that of one (Node 2) was varied as iterations from 10 to $40 \mathrm{GHz}$. [1]

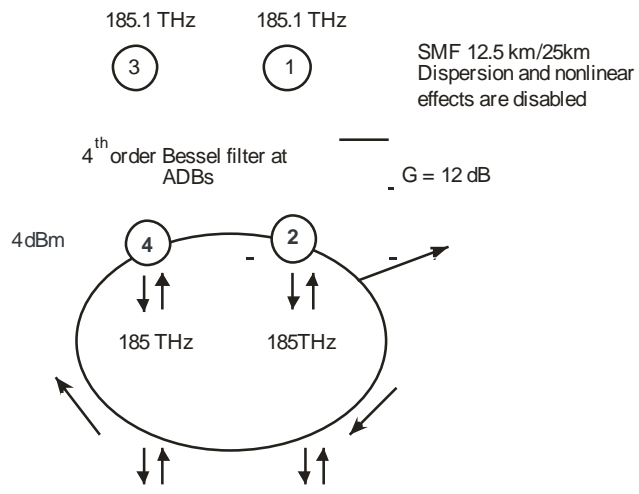

Fig. 2. A ring network with 4 nodes.

The ring network shown in fig. 2 is ended with a control component, the same can circulate the signals in the ring for a $\mathrm{n}$ number of times. The distance between nodes is taken 12.5 and $25 \mathrm{~km}$ and we inserted an ideal amplifier just before node 2 to compensate for the total fiber loss in the ring. The simulation is done at 10,20 , and $40 \mathrm{Gbps}$ at a wavelength of $1652.52 \mathrm{~nm}$, power level $4 \mathrm{dBm}$. The other parameters are Laser line width $1 / 410 \mathrm{MHz}$, Laser noise threshold $1 / 4100 \mathrm{~dB}$, Extinction ratio $1 / 430 \mathrm{~dB}$, responsively $1 / 41 \mathrm{~A} / \mathrm{W}$, Dark current 1/4 $10 \mathrm{nA}$, DGD const. $1 / 43$ ps/km, Birefringence const. $1 / 45 \mathrm{~m}$, Peak Raman gain coiff. 1/4 $9.91014 \mathrm{~m} / \mathrm{W}$, Pump 1 of peak 1/4 $1000 \mathrm{~nm}$, Raman self shift time $1 / 45 \mathrm{P}$ s. Dispersion and nonlinear effects of fibers are disabled to observe the crosstalk effect of ADM [3].

WDM add and drop components are created by using fourthorder Bessel filters. The results for Q factor, eye closure and $\log$ of min BER have been reported for both the cases (12.5 and $25 \mathrm{~km}$ ) by sweeping the bandwidth of filters of one node only from 10 to $40 \mathrm{GHz}$.

The Transceiver Subsystem comprised of a pseudo-random generator which generates bits at $10 \mathrm{Gbps}$, a CW laser as an optical source, a Mach-Zehnder Modulator, a WDM add and drop multiplexer, an attenuator, a PIN diode detector and a fourth-order Bessel low pass filter whose cutoff frequency is 0.80 bit rate.[6].

\section{SIMULATION}

The set up provide how to simulate a Ring $\mathrm{O}$ network with an unrolled equivalent configuration using the iteration feature. A very low noise feeds an iterate block and each span represents a loop cycle. Four spans are used here to have the assessment of the ring optical network. By increasing the number of iterations it is possible to improve the simulation accuracy. Four nodes compose the ring, and at each node a channel is added and another channel is dropped. [8] The analysis of results is made by means of measurement blocks inserted in the iterated block. In this way, it is possible to analyze the signal evolution (spectra, eye diagrams) at each round of the loop. The latest iteration represents the most accurate evaluation of the "steady-state" signal.

The analysis of ring optical network is done by taking four nodes. The node is followed by a fiber of length $50 \mathrm{~km}$ and a splitter. To each node is attached an electrical scope to analyze the signal in terms of $\mathrm{Q}$ factor and jitter.[8] Further, the node is composed of nrz_transmitter, receiver and an optical add drop multiplexer (OADM). At the four nodes the used frequencies are $\mathrm{f} 1, \mathrm{f} 2, \mathrm{f} 3$ and $\mathrm{f} 4$. We have assumed that all these frequencies are used in a circular fashion in the ring, i.e. at node 1 the frequency $\mathrm{f} 1$ is added and the frequency $\mathrm{f} 4$ is dropped.[9]

At node 2 the frequency $\mathrm{f} 2$ is added and the frequency $\mathrm{f1}$ is dropped and so on, which means that at node $\mathrm{n}$ wavelength $\ln$ is inserted and previous node wavelength ln 1 is dropped. Simulation of the RingO optical network is done with the centre frequency of $193.4 \mathrm{THz}$ and the reference bit rate of $10 \mathrm{~GB} / \mathrm{s}$.

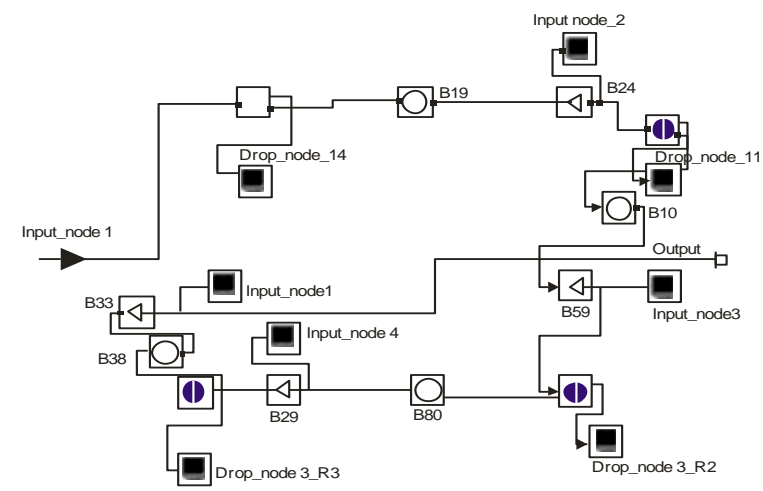

Fig. 3. Set up for ring optical network

Each node is further composed of OADM, one transmitter source and one receiver source. Non-return to zero transmitters is used here. The analysis of results is made by means of measurement blocks inserted in the iterate block. The used frequencies in the ring optical network at the various nodes are, frequency f1 is equal to $193.35 \mathrm{THz}$, frequency f2 is 193.4 and so on, with the difference of .5 THz (Fig. 3).[10]

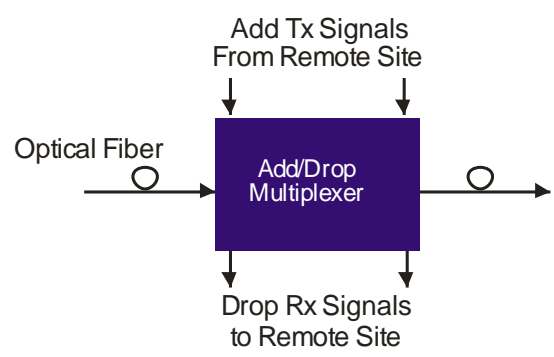

Fig. 4. Optical add drop mux

\begin{tabular}{|l|l|}
\hline Symbol Name & Value $(\mathrm{THz})$ \\
\hline f1 & 193.35 \\
\hline f2 & 193.4 \\
\hline f3 & 193.45 \\
\hline f4 & 193.5 \\
\hline
\end{tabular}

Fig. 5 parameters for ring 
NRZ transmitter

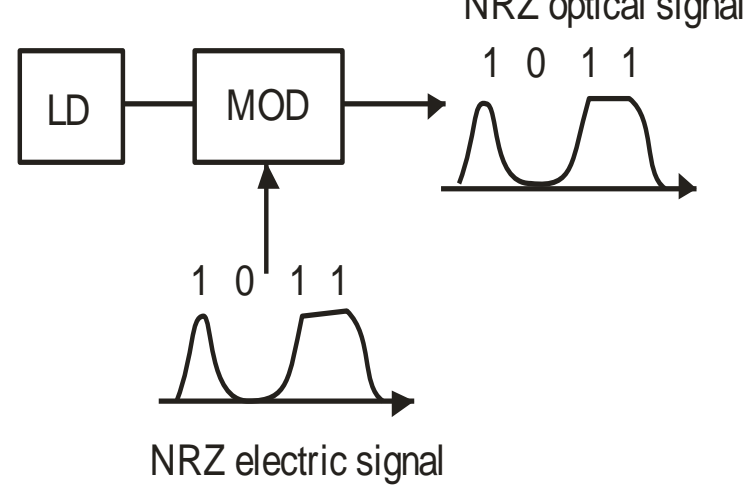

Fig. 6. NRZ transmitter

In OADM, given at the input port a signal, the selected signal is dropped and available at the drop port. Another signal is to be added at the add port. An OADM is a device used in wavelength-division multiplexing systems for multiplexing and routing different channels of light into or out of a SMF. This is a type of optical node, so it is generally used for the construction of a ring-based optical telecommunications network. "Add" and "drop" here refer to the capability of the device to add one or more new wavelength channels to an existing multi-wavelength WDM signal and to drop one or more channels, routing those signals to another network path. An OADM may be considered to be a specific type of optical cross-connect. [11]

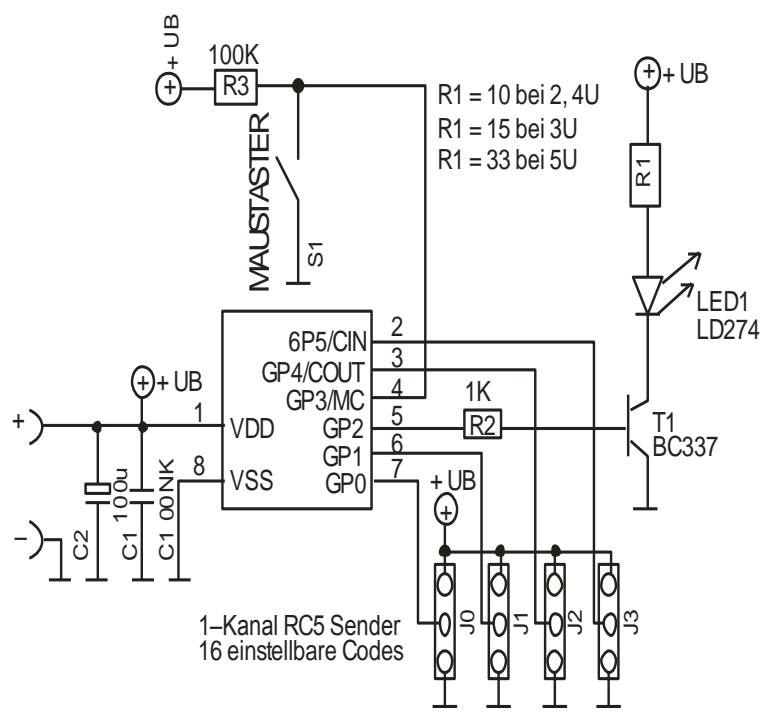

Fig. 7. Channel receiver

The transmitter is composed of data source, laser source, optical amplitude modulator and optical link section. Data source is customized by baud rate, sequence, logical signal level and the period length. In this transmitter set up, the NRZ modulation format is used. The laser source provides transmission at $1552.52 \mathrm{~nm}$ laser emission frequency. Laser phase noise is taken into account by generating a Lorentzian, whose power spectrum width at half maximum is specified at $10 \mathrm{MHz}$.[12] The output from the driver and laser source is passed to the optical amplitude modulator. The optical signal from the modulator is passed through the optical link section composed of SMF and the optical coupler. Single receiver is composed of optical raised cosine filter, PIN photodiode and low-pass Bessel filter. At the receiver measurements are made with the help of an optical spectrum analyzer, optical probe and electrical scope. The electrical scope is used to obtain eye diagram, and from the eye diagram the values of $\mathrm{Q}$ factor, jitter and eye opening are determined. [13]

\section{RESULT}

The results are in the form of eye diagrams from which various signal evolution parameters can be calculated. The eye diagram as shown in Fig. 8 gives the signal at node 1 captured in the first span. At node 1 the jitter is $0.014406 \mathrm{~ns}$ and the average eye opening is $1.66035 \mathrm{e} 015$. The BER is 0.0227501 and the $Q$ value is $6.020600 \mathrm{~dB}$. Since the impact of low-noise source is predominant, these metrics are not yet sufficient for analysis of signal evolution. The simulation bandwidth is from $\quad 1549.11488438 \mathrm{~nm}$ to $1551.11865477 \mathrm{~nm}$. The eye diagram shown in Fig.9 gives the signal at node 2 captured in the first span. [8]

At node 2 the jitter is $0.0240403 \mathrm{~ns}$ and the average eye opening is $1.20984 \mathrm{e} \quad 005$. The BER is $1 \mathrm{e} 040$ and the Q value is $30.359835 \mathrm{~dB}$. It is seen that there is high rise in quality factor of the signal captured in the first span. This is due to the fact that the effect of noise in more on the starting number of users (Fig. 10). There is an improvement in jitter, showing improvement in signal in Fig. 11. [8, 14]

At node 3 the jitter is $0.0238927 \mathrm{~ns}$ and the average eye opening is $1.20706 \mathrm{e} 005$. The BER is $1 \mathrm{e} 040$ and the $\mathrm{Q}$ value is $31.297732 \mathrm{~dB}$. At node $2 \mathrm{Q}$ factor is 30.35 , but here at node 3 it has risen to the value of 31.29 , depicting a further increase in the $\mathrm{Q}$ factor. Thus as the signal passes through the successive nodes improvement in quality factor is seen (Fig. 12).

At node 4 the jitter is $0.02405 \mathrm{~ns}$ and the average eye opening is $1.2062 \mathrm{e} 005$. The $\mathrm{BER}$ is $1 \mathrm{e} 040$ and the $\mathrm{Q}$ value is 32.454378dB as depicted in Fig. 11.

In order to simulate the network the bandwidth of Nodes 1, 3, 4 was fixed at $10 \mathrm{GHz}$ and for Node 2 it was varied as iterations from 10 to $40 \mathrm{GHz}$ :

$\begin{array}{lc}\text { Sweep iteration no. } & \text { Bandwidth }(\mathrm{GHz}) \\ 1 & 10.0 \\ 2 & 17.5 \\ 3 & 25.0 \\ 4 & 32.5 \\ 5 & 40.0\end{array}$

The analysis of results is made by the mean of measurement blocks inserted in the iterated block. Clear observation can be made from the graphs shown in Figs. 8-11, which as the signal passes through every node improvement in the quality factor is seen. The whole ring optical structure is iterated with the four spans. The latest iteration represents the most accurate evaluation of the "steady-state" signal. Also with iteration feature improvement in the quality factor is seen as illustrated in Fig. 12. As is evident from the diagram as the ring optical structure keeps on iterating, its quality factor is also improving. With every span improvement in the quality factor is seen. 


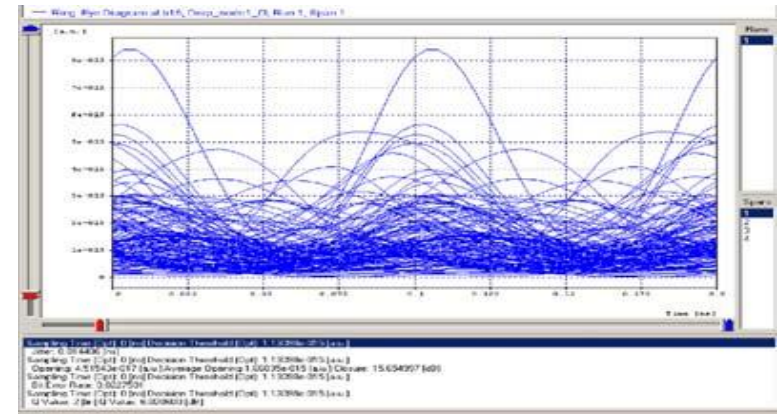

Fig. 8. Eye diagram for node 1.

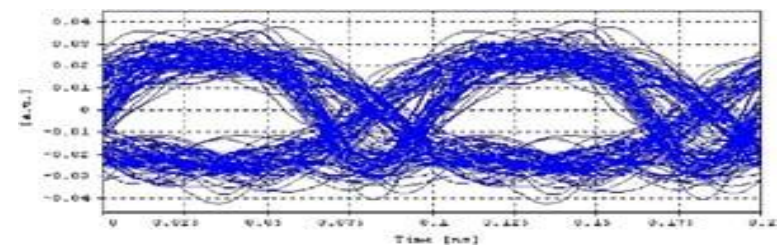

Fig. 9. Eye diagram for node 2.

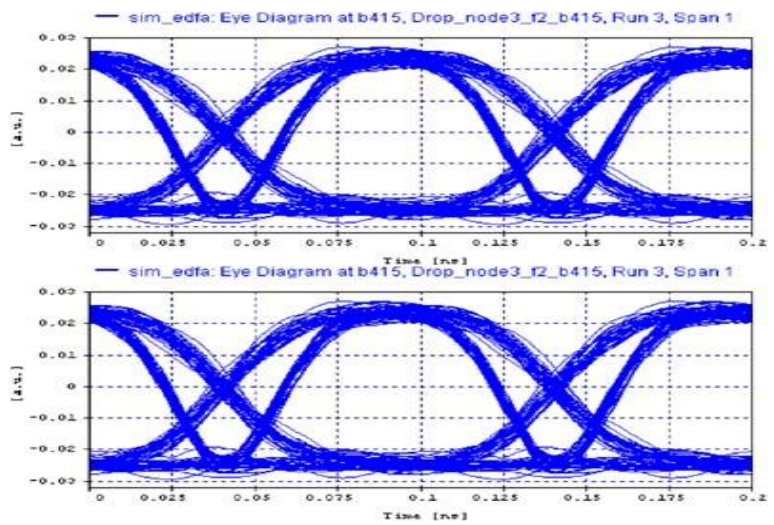

Fig. 10. Eye diagram for node 3.

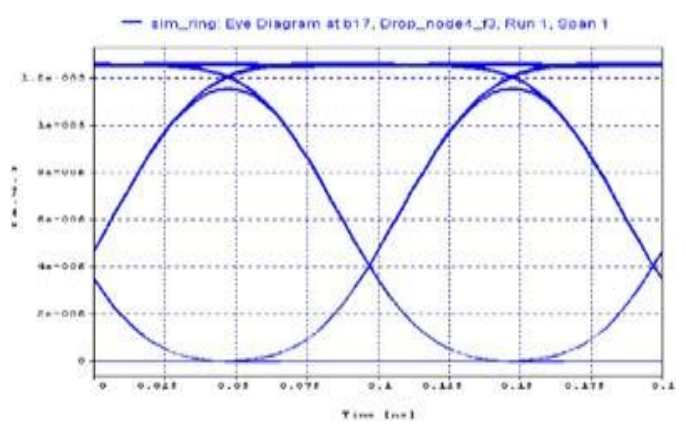

Fig. 11. Eye diagrams at node 4

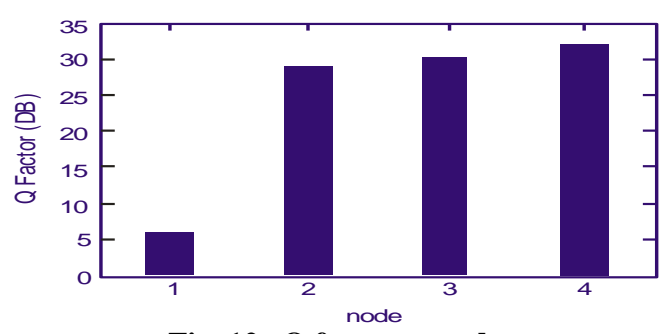

Fig. 12. Q factor vs.node.
At every OADM, one frequency is dropped and other added. This is clear from the optical spectrum taken at every node. As shown in Fig. 13, at node 1 the frequency f1 193.35 is added and the frequency f4 193.5 is dropped. It is assumed that all these frequencies are used in a circular fashion in the ring i.e. at node 1 the frequency $\mathrm{f} 1$ is added and the frequency $\mathrm{f} 4 \mathrm{is}$ dropped. At node 2 the frequency $\mathrm{f} 2$ is added and the frequency $\mathrm{f} 1$ is dropped and so on, which means that at node $\mathrm{n}$ wavelength $\ln$ is inserted and previous node wavelength $\ln 1$ is dropped. Here at node 2 the frequency f2 193.4 is added and the frequency f1 i.e. 193.35 is dropped as shown in Fig. 14. Similarly at node 3 the frequency $\mathrm{f} 3$ is added and the frequency $\mathrm{f} 2$ is dropped. Now at node 4 the frequency f 4 is added and the frequency $\mathrm{f} 3$ is dropped.

If we want to increase the number of nodes in the network, the circle goes so on, i.e. add the frequency fn at node $\mathrm{n}$ and drop the frequency fn 1 of the previous node (Fig. 15 and16).

It is observed that if as we keep on increasing the signal input power, improvement in received optical power is observed. As the input power increases, the output power also increases for all the users. The power penalty goes on increasing with the increase in the signal input power.

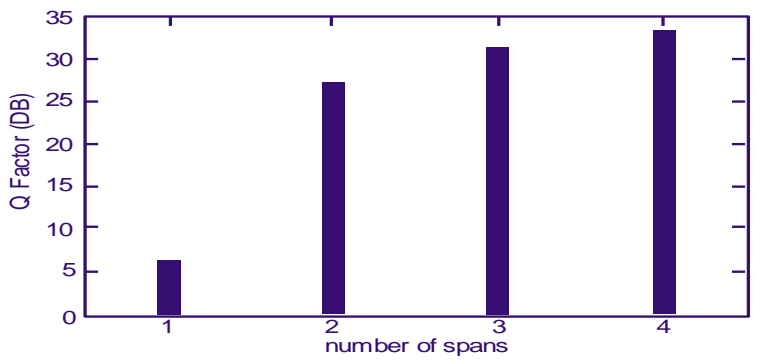

Fig.13. Q factor vs. span number.

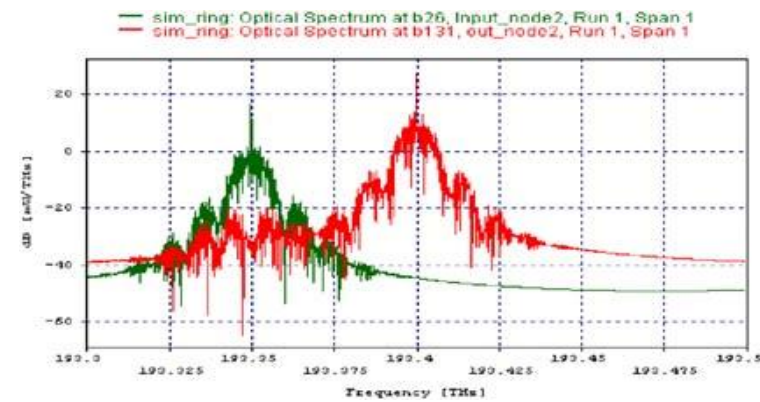

Fig. 14. Optical spectrum at node 1 before and after transmission.

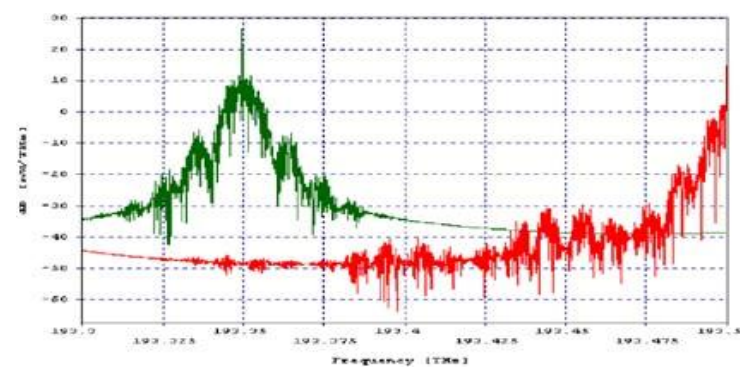

Fig. 15. Optical spectrum at node 2 before and after transmission. 
At low value of signal input power $20 \mathrm{dBm}$ or below, the effect of amplified spontaneous emission (ASE) noise power is quite high [16]; therefore, the quality of signal goes on decreasing. At high value of signal input power 10 $\mathrm{dBm}$ or above, the gain fluctuation occurs at high rate (Fig. 17).

Therefore, gain drops causes power penalty. The power value should neither be so high nor so less because with high power the degradation in performance through self-phase modulation can occur. On the other hand, too little power results in not enough gain. For the low signal input power of $40 \mathrm{dBm}$, the received power is very less. But as the input power keeps on increasing up to $0 \mathrm{dBm}$, the received power also increases. Hence it can support more number of users by increasing the signal input power up to a certain range. This is because with the increase in input power, the output received power also increases. By increasing the input power from 5 to $0 \mathrm{dBm}$ and onwards, the input power increases in excess and saturation is reached, and thus gain starts decreasing and the gain of the amplifier reduces. This effect is known as gain saturation signal. [8, 15]

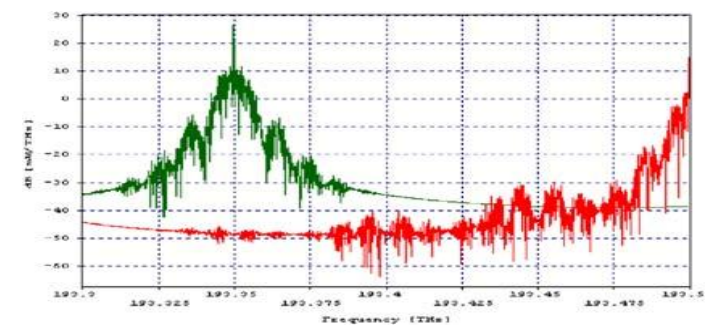

Fig.16. Optical spectrum at node 4 before and after transmission

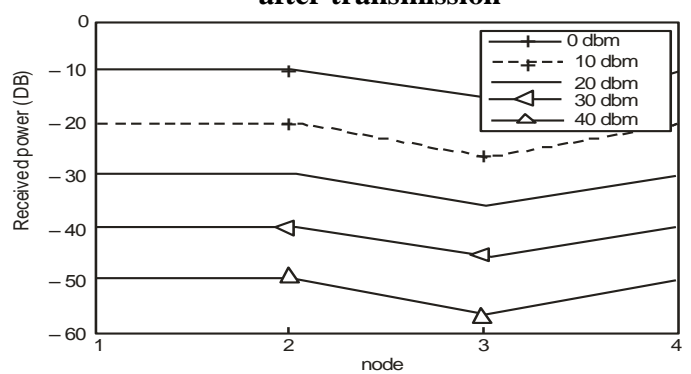

Fig. 17.Received power at different input powers

But input power cannot be increased to a high value as the device structure imposes a limit on the maximum high input power.

OSNR is one of the most important parameters of the signal analysis. It is the ratio of signal power to the noise power corrupting the signal. Its value should be high enough to ensure desired signal output. The OSNR increases with the input power of the signals launched into the transmission line. However, high input power may lead to increase in the influence of saturation effects as well as nonlinear effects of the fiber. Hence, a trade-of exists between the OSNR after the transmission line and saturation effects in the optical amplifier as well as nonlinear effects in the fiber ASE noise contributes not only to the OSNR reduction but also to the saturation, leading to decline in gain. Here as shown in Fig. 18, OSNR is increasing as we move from node 1 to node 4 in the ring. This also depicts that the signal quality is improving as the number of node increases. But after certain nodes its value drops due to saturation (Fig. 19). [8]

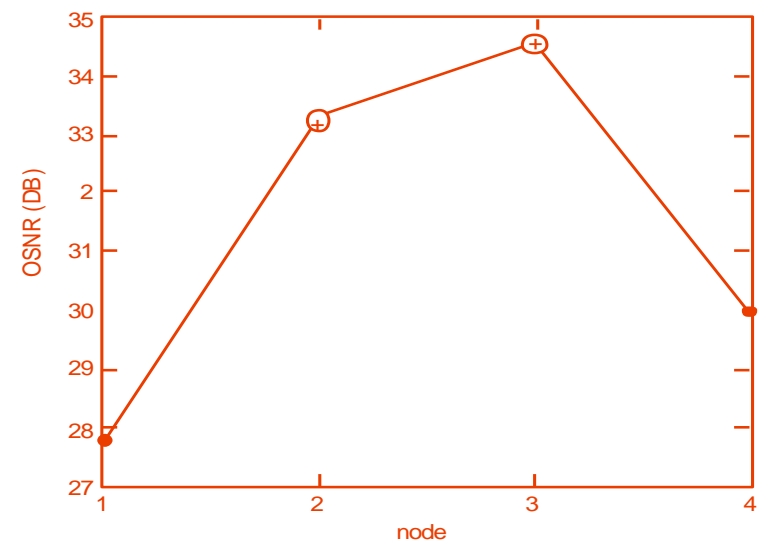

Fig. 18. Optical signal to noise ratio at different nodes

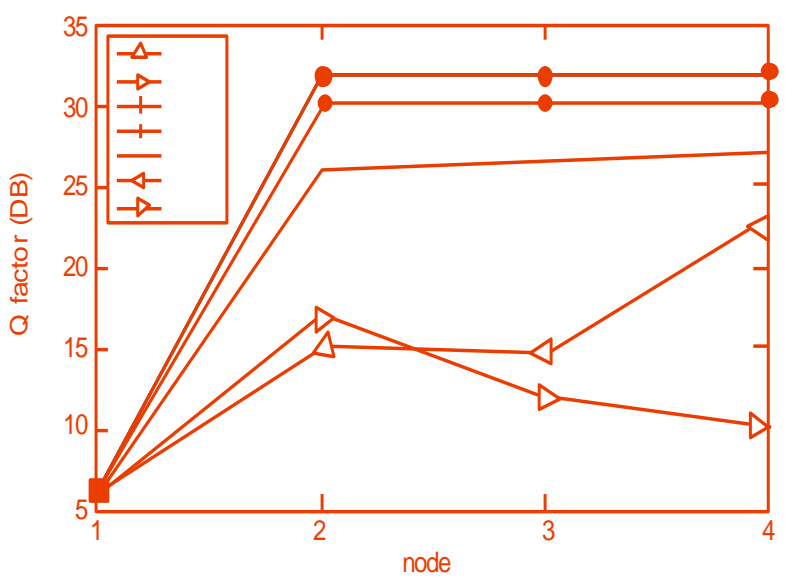

Fig. 19. $Q$ factor variation for various lengths

Optical fiber is generally chosen for systems with higher bandwidths or spanning longer distances than electrical cabling can provide. In the long-distance network, the majority of embedded fiber is standard single mode (G.652) with high dispersion in the 1550-nm window, which limits the distance for transmission. Dispersion can be mitigated to some extent and at some cost using dispersion compensators. Non-zero dispersion-shifted fiber can be deployed for transport, but higher optical power introduces nonlinear effects. In the short-haul network, PMD and nonlinear effects are not so critical as they are in long-haul systems, where in higher speeds (OC-192 and higher) are more common. First we simulate our model taking fiber lengths of 50 and $100 \mathrm{~km}$. Effect of fiber length on the Q factor wrt to various spans is not much. But as simulation is carried out for the various lengths of fiber, it is seen that we can increase the fiber length up to maximum $250 \mathrm{~km}$, after that its Q factor keeps on deteriorating. Except for the first span, not much improvement in the $\mathrm{Q}$ factor is seen, but as the fiber length is increased, improvement can be seen for fiber length up to a certain range. 


\section{CONCLUSION}

Ring networks can be used to provide a cost effective and an easily implementable way of achieving WDM networks. The coverage of metro area network is possible as ring topology is the main backbone among metropolitan cities. Here in this paper, the analysis of the ring optical network is done by taking four nodes. The signal is analyzed as it passes through each node in the ring optical network.

It can be concluded from eye diagrams and jitter values taken at every node that there is no appreciable signal degradation in the ring network. It is seen that there is an increase in the quality factor i.e. signal keeps on improving as it passes through the successive nodes. This is one of the main advantages of RingO networks. As signal goes from node 1 to node 2 its quality factor value changes from 6.02 to 30.02 . When the whole ring structure is iterated with the help of spans, then improvement in the signal value is seen, as it passes through the various spans. Analysis of the $\mathrm{Q}$ factor is done taking different lengths of the fiber. It is seen that there is no appreciable signal degradation till the length of the fiber is increased up to $250 \mathrm{~km}$. But after that a decrease in the quality factor can be seen. [8]

For the low signal input power of $40 \mathrm{dBm}$, the received power is very less. But as the input power keeps on increasing up to $0 \mathrm{dBm}$, the received power also increases. Hence it can support more number of users by increasing the signal input power. This is because with the increase in input power, the output received power also increases. By increasing the input power from 5 to $0 \mathrm{dBm}$ and onwards, the input power increases in excess and saturation comes thus gain starts decreasing and the gain of the amplifier reduces. Optical spectrum of the signal is seen at every node, to have the assessment of the add and the drop frequencies in the ring. Thus, complete analysis of the ring optical network is done here, which proves to be beneficial for the deployment of ring as the main backbone in our present infrastructure.[8]

The performance of the ring network varied with each sweep iteration, i.e. as the bandwidth of the WDM add and drop multiplexer was increased from 10 to $40 \mathrm{GHz}$ in steps, the Q factor of the system, the eye opening increased while the minimum BER was further reduced. The crosstalk on the other hand increased. With increase in bit rate (speed), the performance of the ring optical network deteriorated. With 10 Gbps the network was more immune to crosstalk than 20 or 40 Gbps. Also with increase in length of the optical fiber connected between the nodes, crosstalk and the dispersion effects became more prominent. For a $12.5 \mathrm{~km}$ fiber length system the eye opening was much wider as compared to that in the $25 \mathrm{~km}$ fiber length network. [1]

\section{REFERENCES}

[1] Vinod Kumara, Ajay K. Sharmab, R.A. Agarwalac "Investigation on interchannel crosstalk at for an optical ring network". Optik 121 (2010) 45-49
[2] G.P. Agrawal, Fiber Optic Communication System, Wiley-interscience, New York, 1997.

[3] Tim Gyselings, et al., Crosstalk analyses of multiwavelength optical cross connects, J. Lightwave Technol. 17 (1999) 1273.

[4] R. Ramaswami, K.N. Sivarajan, Optical Networks: A Practi-cal Perspective, Morgan Kaufmann, Los Altos, CA, 1998

[5] G.R. Hill, et al., A transport network layer based on optical network elements, J. Lightwave Technol. 13 (1995) 841-849.

[6] E.L. Goldstein, L. Eskildsen, A. Elrefaie, Performance implications of component crosstalk in transparent lightwave networks, IEEE Photon. Technol. Lett. 6 (1994) $657-659$.

[7] Optical add-drop multiplexer - Wikipedia: http://en.wiki pedia.org/wiki/Optical_add-drop_multiplexer.

[8] Rajneesh Randhawaa, , J.S. Sohalb, R.S. Kaler, Optimum performance and analysis of RingO networks.

[9] Cheoul-Shin Kang, Byung-Seok Park, J. ong-Dug Shin, J. e-Myung Jeong, A broadband ring network: multichannel optical slotted ring, Comput. Networks ISDN Syst. 27 (9) (1995) 1387-1398.

[10] R. Gaudino, A. Carena, V. Farero, A. Pozzi, F. Neri, P. Poggiolini, RingO: A WDM ring optical packet demonstrator, in: Proceedings of the 27th European Conference on Optical Communication, Amsterdam,2001.

[12] J. Cole Smith, Algorithms for distributing telecommunication traffic on a multiple-ring SONET-based network, Eur. J. Oper. Res. 154 (3) (2004) 659-672.

[13] T.-H. Wu, R.C. Lau, A class of self-healing ring architectures for SONET network applications, in: Proceedings of the IEEE GLOBECOM '90, San Diego, CA, December 1990, pp. 444-0451.

[14] M.I. Irshid, M. Kavehrad, A fully transparent fiber optic ring architecture for WDM networks, IEEE J. Lightwave Technol. 10 (1992) 101-108.

[15] W.I. Way, D.A. Smith, J.J. Johnson, H. Izadpanah, A self-routing WDM high-capacity SONET ring network, IEEE Photon. Technol. Lett. 4 (1992) 402 405 .

[16] H.V. Madhyastha, G.V. Chowdhary, N. Srinivas, C. Siva Ram Murthy, Grooming of multicast sessions in metropolitan WDM ring networks, Comput. Networks 49 (4) (2005) 561-579.

[17] Yong $\mathrm{Xu}, \quad$ Shen-Chu $\mathrm{Xu}, \mathrm{Bo}-\mathrm{Xi} \mathrm{Wu}$, Traffic grooming in unidirectional WDM ring networks using genetic algorithms, Comput. Commun. 25 (13)(2002) $1185-1194$. 